\title{
Customized Mobile Marketing Platform Design Utilizing IoT based Beacon Sensor Devices
}

\author{
Ji-Hoon $\mathrm{Seo}^{1}$ and Jin-Tak Choi ${ }^{2}$ \\ ${ }^{1}$ Department of Computer Engineering, Incheon National University, South Korea \\ ${ }^{2}$ Department of Computer Engineering, Incheon National University, South Korea \\ ${ }^{1}$ sserz@inu.ac.kr, ${ }^{2}$ choi@inu.ac.kr
}

\begin{abstract}
As diverse mobile devices such as smartphones and tablet PCs are rapidly popularized throughout the world, models for providing diverse services to users through these portable terminals have been rapidly appearing. In recent years, online marketing service techniques have been appearing with which Beacons, which are low-power Bluetooth protocol based short-range wireless communication devices, are installed in those types of shops such as marts, shopping malls, and department stores to monitor whether users have visited the relevant shops in real time through communication with the mobile devices of customers, who are clients, and when customers are judged to have visited the relevant shops, the customized advertising information and coupons based on the customer profiles are transmitted to the mobile devices of the customers. The most common technique among them is to transmit e-coupons for diverse products to the customers' mobile devices, thereby inducing the customers to use the relevant e-coupons. However, the marketing service that utilizes the customer information obtained through the transmission and reception of e-coupons that have been already issued is operated in the form of transmitting e-coupons in a random manner without considering the customers' shop visit patterns. For this reason, cases where the weak point of marketing is exposed so that even if e-coupons are transmitted to customers' mobile devices, the relevant customers do not revisit the shops occur frequently. Therefore, in this paper, a solution will be presented for more effective mobile marketing service platforms and operation algorithms that can properly transmit and provide customized e-coupons to the relevant customers by analyzing the profile information and patterns of customers who received the coupons transmitted through Beacon sensors.
\end{abstract}

Keywords: Beacon, mobile marketing service, big data, customer visit pattern

\section{Introduction}

Currently, using mobile devices that have been popularized throughout the world including not only advanced countries but also developing countries with rapidly growing economies centering on the IT technology as mediators, the app content industry has been growing remarkably in diverse fields including mobile marketing services for the culture, arts, humanities, engineering, and education in the overall society. In line with the foregoing, diverse kinds of mobile marketing services are currently being provided in South Korea too, and among them, online marketing services using low power shortrange wireless communication networks are attracting public attention. Recently, diverse short-range wireless communication network technologies such as RFID, QR Code, NFC, and BLE have been developed and released. Among them, a technology that is becoming an issue is the Bluetooth Low Energy (BLE) technology. This BLE has emerged as an issue of the short-range wireless communication network technology along with Beacon

Received (November 29, 2017), Review Result (January 20, 2018), Accepted (January 29, 2018) 
sensor devices. Beacons have a technique to enable service providers to transmit ecoupons related to diverse products to customers' smartphones thereby inducing users to use the relevant e-coupons [1] [2]. The mobile marketing service technology utilizing low power Bluetooth based beacon sensor devices contributes to the diffusion of diverse portable terminals including smartphone. However, most of the mobile marketing services currently provided are operated in the form of issuing e-coupons through random transmission without considering customers' shop visit patterns so that most of the relevant users recognize the e-coupons as spam messages and do not revisit the relevant shops[3]. In addition, the field of short-range wireless communication technologies, which has achieved rapid progress thus far, has technological limitations in that applicable functions are limited because of temporary storage spaces and constraints on the distances to service areas and that qualitatively poor data information is handled. Therefore, in this paper, an integrated platform construction model and an operation algorithm for online marketing services based on the low power Bluetooth Beacon technology will be presented [4]. In addition, utilizing the Beacon sensor technology, the coupon transmission method for existing online marketing services that is for just one-off transmission will be developed one step further to contribute to next generation urban marketing conversion service strategies that will enable substantial and continuous provision of marketing services through transmission of customized coupons based on statistical analysis of big data containing customer information collected focusing on customer follow-up management.

\section{Related Work}

The beacon used in this study is a wireless communication device that can transmit related information to mobile devices detected within a short distance (about $50 \mathrm{~m}$ ) by using the low power Bluetooth (BLE) technology.
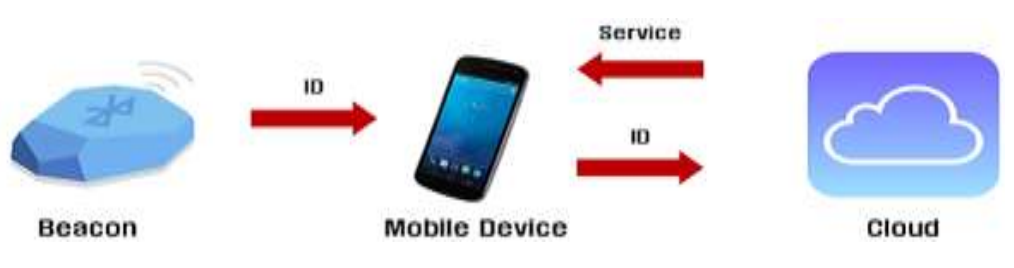

Figure 1. Operating Concept of Beacon

Recently, low power Bluetooth (BLE) technology based Beacons with low battery consumption as with the iBeacon ${ }^{\mathrm{TM}}$ have been emerging as the mainstream. Beacon services operate through a way in which smartphone apps receive beacon signals and transmit the signals to the dedicated server, and the server identifies the information and displays the information on the apps.

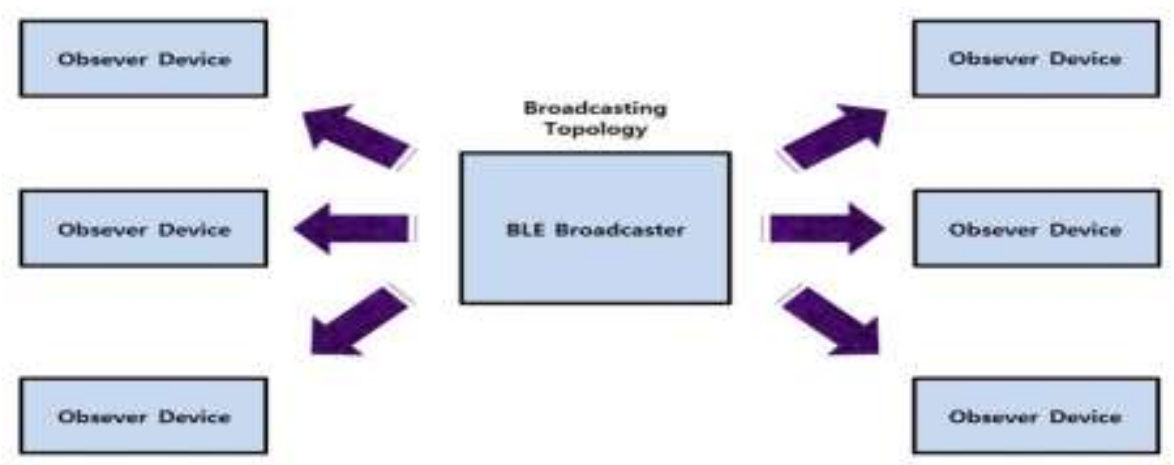

Figure 2. BLE Broadcaster's Broadcasting Topology 
If a beacon is installed in a shop, the beacon can be utilized as a useful promotional means for product information dispatch and coupon issuing [5]. In addition, the installation of a beacon is advantageous for the creation of diverse services because the location information service can be provided by inputting the latitude and longitude information into the beacon [6]. Since beacons automatically find the locations of customers and provide information or services, the convenience of use is increasing from the viewpoint of and beacons are attracting attention as a new core technology in the mobile payment market [7]. Thus far, a representative technology in the mobile payment market has been relevant to NFC. Although NFC is highly safe in terms of security because payments are made only when the terminal should come into contacted with the payment device within $\mathrm{n}$ a distance not farther than $10 \mathrm{~cm}$, it has a disadvantage of requiring the installation of a very expensive NFC reader [8][9]. On the contrary, beacons do not require 'contact' because payments are made through the customer information registered by the user in the smartphone app and beacons are quite advantageous from the viewpoint of shops in terms of costs because shops may install beacons, which are relatively cheap, instead of the expensive NFC readers [10][11] Recently, the importance of beacons as customer contact points for information collection and service provision has been increasing as their use has been rapidly expanding for marketing for offline sales improvement to financial services and services utilizing spatial information such as theme parks [12-14].

Table 1. Beacon vs. NFC Comparison

\begin{tabular}{|c|c|c|}
\hline Device & Beacon & NFC \\
\hline Technology & $\begin{array}{l}\text { Bluetooth low energy } \\
\text { (BLE) }\end{array}$ & $\begin{array}{l}\text { Radio frequency } \\
\text { identification } \\
\text { (RFID) }\end{array}$ \\
\hline Range & Up to 50 meters & Up to $20 \mathrm{~cm}$ \\
\hline Accuracy & 2-3 inches & Very accurate \\
\hline $\begin{array}{l}\text { Energy } \\
\text { efficiency }\end{array}$ & Very efficient & $\begin{array}{l}\text { Consumers relatively } \\
\text { more power than BLE }\end{array}$ \\
\hline Payment & Compatible & Compatible \\
\hline Cost & Affordable & Might be costly \\
\hline Security & Relatively vulnerable & Relatively excellent \\
\hline $\begin{array}{l}\text { Users' } \\
\text { demographics } \\
\text { and trust }\end{array}$ & $\begin{array}{l}\text { Smart phone users are } \\
\text { very affluent }\end{array}$ & $\begin{array}{l}\text { Lack of awareness and } \\
\text { trust }\end{array}$ \\
\hline Features & $\begin{array}{ll}\text { - } & \text { 1:n service centric } \\
\text { - Various software } \\
\text { - Active }\end{array}$ & $\begin{array}{ll}\text { - } & 1: 1 \text { service centric } \\
\text { - Insufficient software } \\
\text { - Passive }\end{array}$ \\
\hline
\end{tabular}




\section{Proposed Method}

\subsection{Major Operation Algorithms for User Customized Marketing Service Devices}

In this study, algorithms are presented based on customized marketing services that include two information receiving parts; one counting part for counting the number of times of receipt of information identifying the records of visits of customers generated by each of multiple affiliated terminals existing at individual shops whenever the smartphone of an app service customer is accessed by the beacon installed in each shop and an ecoupon issuing part that creates e-coupons for products designated as products for which e-coupons should be issued by one or more shops and transmits the e-coupons to the smartphones of app service customers.

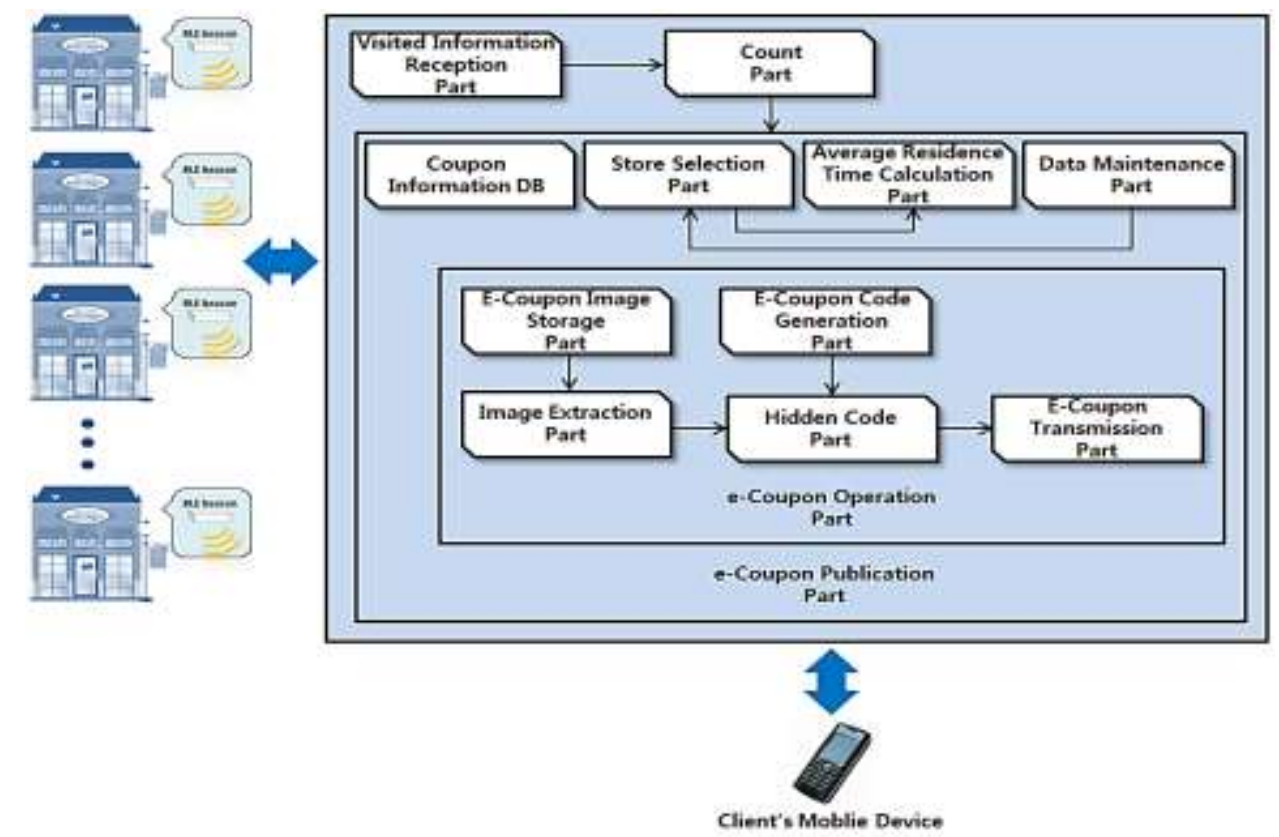

Figure 3. Block Diagram of the Beacon Operation Process for Customized Mobile Marketing Service

The information receiving parts composed of these two parts receive the customer visit identifying information generated by individual terminals from at least one affiliated shop and store the customer profiles such as the age, gender, and occupation, etc., collected when the customer first registered his/her account including the stay time of the customer into the database on receipt of the information to exchange the information with each other through the main server system and the cloud server.

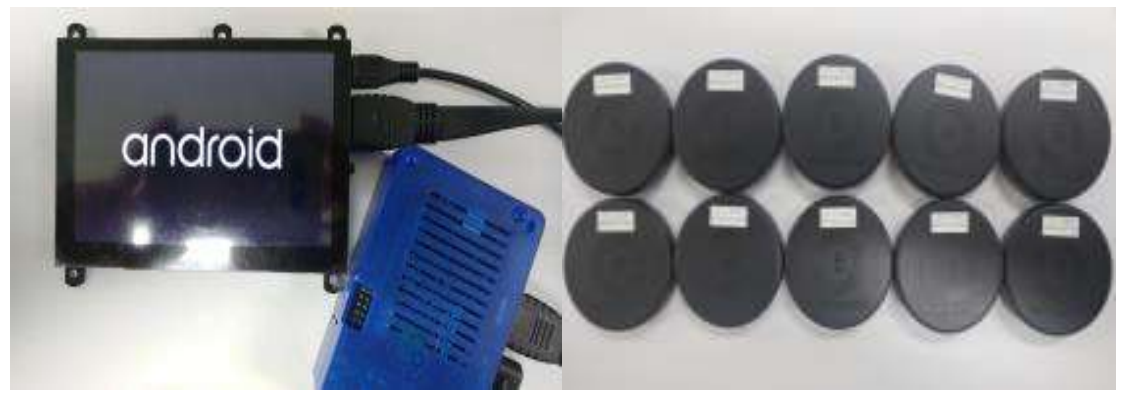

Figure 4. Odroid-C2 (Left) and Onyx Beacon One (Right) used for Device Function Test 


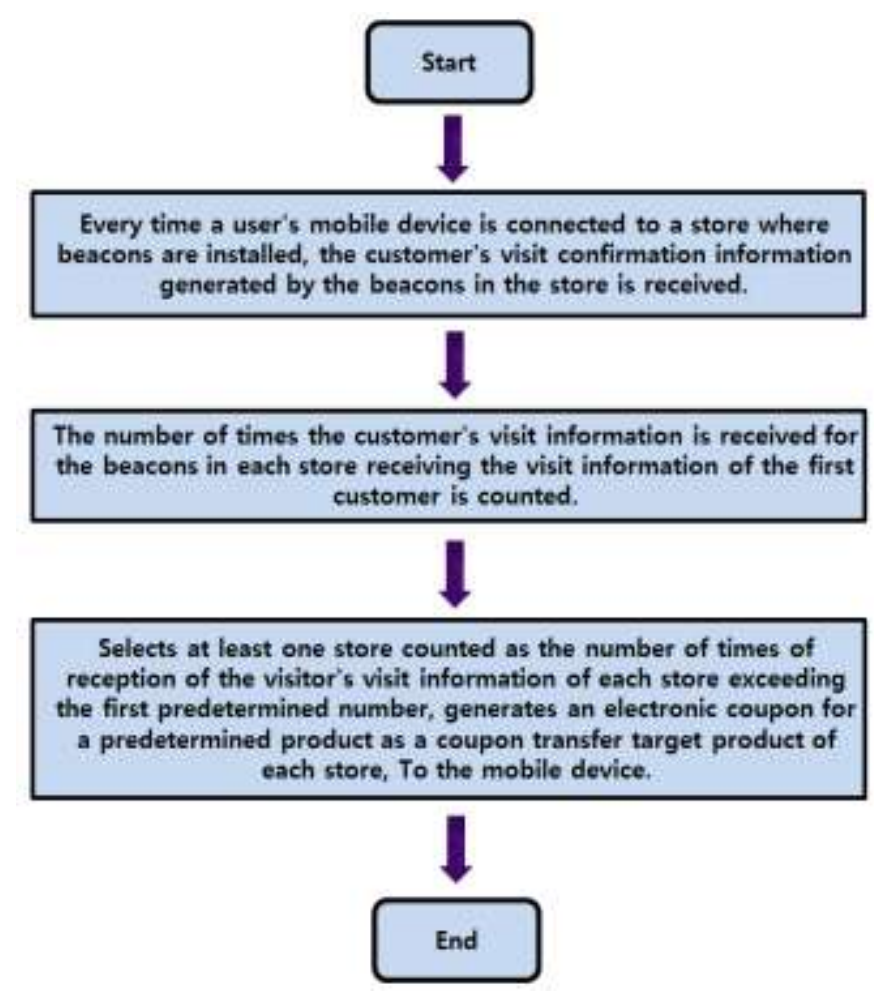

\section{Figure 5. Beacon Operation Algorithm for Customized Mobile Marketing Service}

The counting part counts the number of times of receipt of customer visit identifying information from each affiliated shop while recording the total stay time of customers and the e-coupon issuing part is in the form of a coupon information management system in which the pieces of information on products, which are the targets of coupon issuing, are stored for different stay time ranges consisting in multi-steps for each shop.

\subsection{Major Components of the App that is Interlocked with the Low Power Bluetooth Beacon Sensor Device}

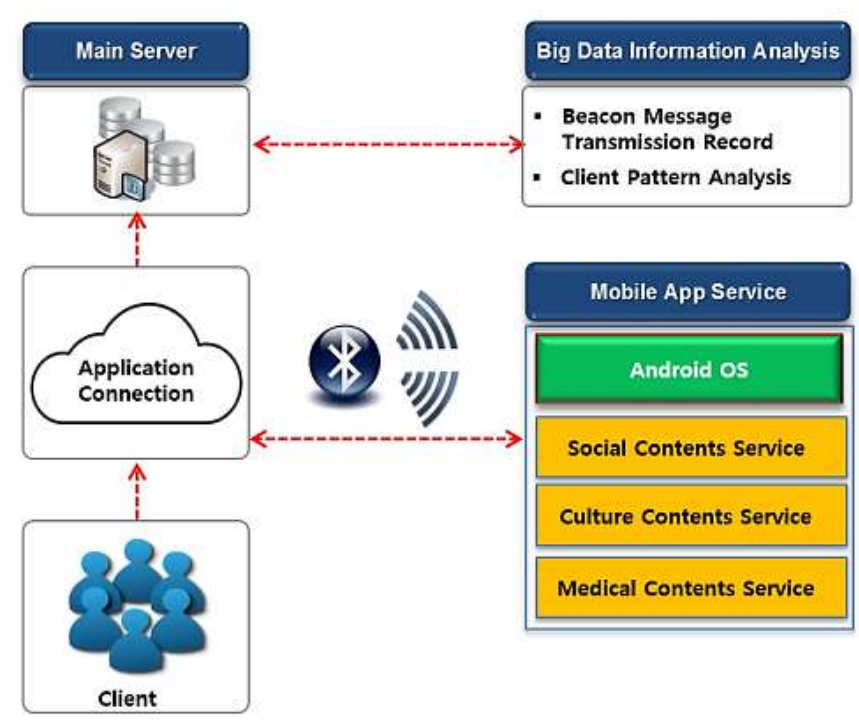

Figure 6. Block Diagram of the Mobile Marketing Service System 
In this study, the app content dedicated to the beacon, which is interlocked with low power Bluetooth Beacon sensor devices was divided into three areas; that is, social, cultural, and medical contents.

\subsection{Broadcast Technique for Identification of Beacon Apps}

In this study, the basic elements of UUID, major and minor were utilized as broadcasting elements for identification of beacon sensor device apps. The universal unique user identifier (UUID) is used as a key value to identify the visit records of authenticated clients and is usefully utilized for app construction. In general, the use case quantitative sub-range is allowed. The major value is the value specifying the specific beacon and the use case. In this study, the major value was designated as a certain beacon and the minor values, which are values to designate use cases, were designated as the inner compartments of the actual shop where the beacon sensor device was constructed, seats in the stadium, or certain areas in the exposition.

Table 2. Beacons Use Case Table's Development

\begin{tabular}{|l|l|l|l|}
\hline \multicolumn{2}{|l|}{ Corner } & Foods & Petticoat \\
\hline UUID & 8F0C1DDC-11E5-4A07-8910-425941B072F9 \\
\hline Major & 1 & 2 \\
\hline \multirow{3}{*}{ Minor } & 1 & $30 \%$ discount event of sushi & $\begin{array}{l}\text { 50\% off all women's } \\
\text { wear }\end{array}$ \\
\cline { 2 - 4 } & 2 & $1+1$ event of pizza & N/A \\
\hline
\end{tabular}

\subsection{Unique Identifier Authentication}

Since low power Bluetooth based Beacon sensor devices have only the function to transmit has the data information received from the main server to the user's mobile device because of their characteristics, their mobile marketing services are limited. To compensate for this limitation, in this study, a technology was introduced that can manage transmission data for individual users through separate servers by applying authentication modes unique to users and construct big data analysis processes to maintain and develop future follow-up management systems for app users based on repetitive analyses of user patterns accumulated by ID.

\subsection{Activity Design Models by Function of App Systems}

In this study, the contents of mobile marketing services apps were divided into 3 areas as shown in the following "Table 3".

Table 3. Functional Activity Design

\begin{tabular}{|l|l|l|}
\hline Society contents & Culture contents & Medical information \\
\hline Goods information & Ticket information & Surgical information \\
\hline Coupon information & Seating information & Staff information \\
\hline Sales information & Match information & Promotion information \\
\hline LBS information & LBS information & LBS information \\
\hline
\end{tabular}

Through the composition of product information, coupon information, sale information, and position based service based admission information that can be utilized in ubiquitous system based daily life, the smart society content becomes to broadcast the 
relevant information when the user has approached the beacon sensor device. The smart culture content is a culture and art convergent content that is composed of components that will enable the user to be provided with information services for stadiums, expositions, and performances through the beacon transmission system and the data that can be received by the user include food corner information, seat information, and ticket information. The smart medical care content enables the user to receive data such as the medical care system, indoor based position information that can be utilized inside the hospital, and medical worker information.

\subsection{Construction of Content Management System (CMS)}

An independent content management system (CMS) is indispensable for more efficient maintenance and management of contents provided by mobile marketing services utilizing low power Bluetooth based Beacon sensor devices. Therefore, in this study, a content management system that can comprehensively manage the plane layout in the shop with beacon sensor devices, battery life, coupon management, customer information collection, and customer visit pattern analysis was constructed. To test the performance of the Beacon sensor devices, a total of five Beacon sensor devices input with different mission codes were installed in the indoor of a building of a university selected for the test in this study. The following the "Figure 7" shows a plane layout of the first beacon sensor device on a real open street map. The plane layout shows a view of the heat map of the beacon sensor device in operation after coming into contact with an actual mobile device installed with the app after the construction of the beacon sensor device.
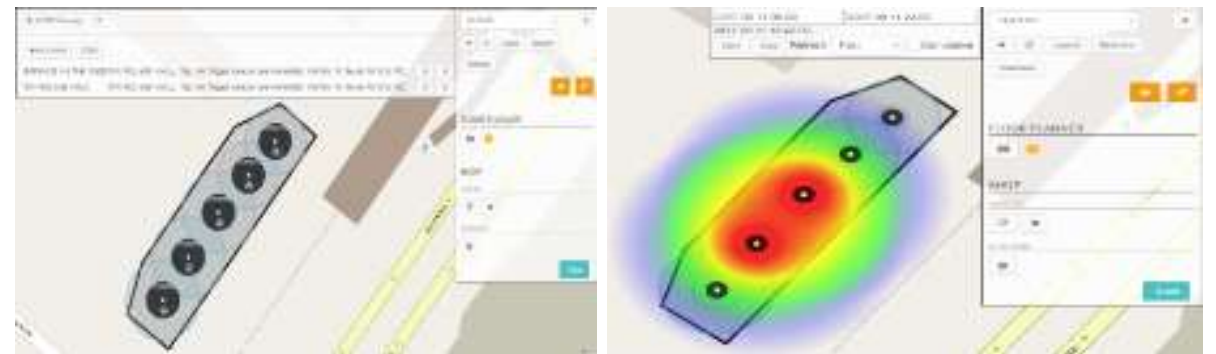

Figure 7. A View of the Plane Layout of a Beacon (Left) and the Heat Map (Right) in Operation in the CMS Platform

\subsection{Active User Information Analysis Beacon Sensor Device Design}

Table 4. Beacon Device's Scanner DB Code

\begin{tabular}{l}
\hline Scanner DB code \\
\hline \hline \\
<provider android:authorities="your.package.name.provider" \\
android:name="com.onyxbeaconservice.provider.IBeaconProvider" \\
android:enabled="true" android:exported="true"> \\
$</$ provider>
\end{tabular}

In this study, to implement more effective mobile marketing services, the importance of the operation algorithm of the beacon sensor device that directly comes into contact with customers' mobile devices to receive information and deliver the information was brought into focus. The beacon sensor device operation algorithm was divided into largely three parts. 
Table 5. Customized Mobile Marketing Service's Beacon Operation Process

\section{1 to 3 step operation process of beacon devices}

\section{START}

STEP 1. This is a shop visit information receiving part in which the beacon sensor device of each shop receives the customer ID and shop visit information whenever the customers' mobile device corresponding to a client closely approaches a shop installed with a beacon sensor device.

STEP 2. This is a count part in which the number of visits to customer's shop visiting information from the beacon sensor device of each shop. An ecoupon issuing part was set to select at least one shop from which the number of times of receipt of customer visit information has been already counted to exceed the first set count among various shops, generate e-coupons for products designated as contents for which coupons should be issued by the relevant shop, and transmit the coupons to the mobile devices of the relevant customers. In addition, the operation algorithm of the customized mobile marketing service device for shop visits corresponds to the part to receive the customer ID and shop visit information data received by the beacon sensor device in the shop from individual mobile devices.

STEP 3. This is a part to collect the databases received from various shops to count the number of times of receipt. That is, this is an e-coupon transmission part to select at least one shop from which the number of times of receipt of customer visit information has been already counted to exceed 1 among various shops installed with beacon sensor devices, generate e-coupons for products designated as contents for which coupons should be issued by the relevant shops, and transmit the coupons to the mobile devices of the relevant customers.

END.

\subsection{Code Hiding System}

The code hiding system is divided into 16 blocks, and each bit value constituting the 16-bit electronic coupon code generated in the electronic coupon code generation part mentioned above can be hided in each of the 16 blocks by changing the pixel values of the multiple pixels existing in each of the 16 blocks according to the pixel value change pattern selected based on the bit values of the electronic coupon code to be hidden in the individual blocks. If the pixel value change pattern selected as mentioned above requires to high a bit value of "1" in a certain block, it will be a pattern to reverse the last four bit values in the pixel value data of the multiple pixels included in the relevant block and if the pattern requires to high a bit value of "o" in a certain block, it will be a pattern to maintain the bit values in the pixel value data of the multiple pixels as they are. That is, if the electronic coupon code is "1011101111110000", the last four for bit values in the pixel value data can be reversed for the 1st, 3rd, 4th, 5th, 7th, 8th, 9th, 10th, 11th, and 12th blocks of the 16 blocks in the code hiding system and can be maintained as they are for the 2nd, 6th, 13th, 14th, 15th, and 16th blocks. Here, the reversal of the last four bit values in the pixel value data means that, if the pixel value data is "11100011", the relevant data will be changed into "11101100". 


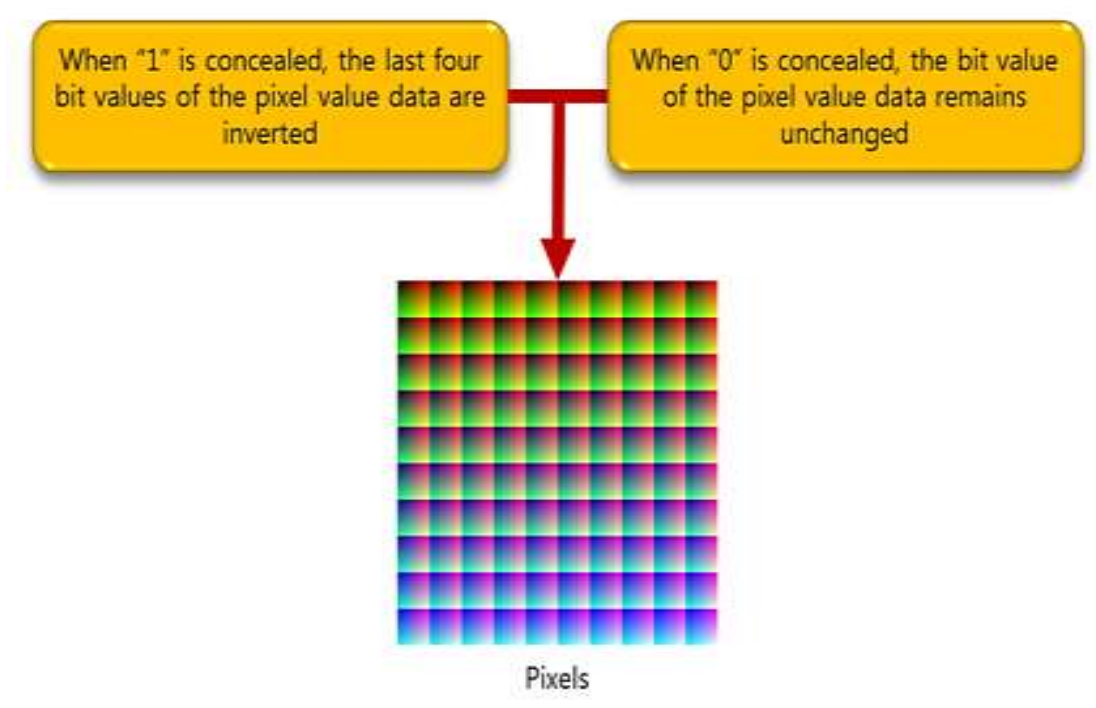

Figure 8. Beacon's Code Hiding Algorithm for Mobile Marketing Service

When the electronic coupon code hiding has been completed through the code hiding system, the electronic coupon transmission system can transmit the electronic coupon for "shop 1" to the mobile terminal of the user. At this time, the mobile terminal of the user stores the same data as shown in the "Table 3" below it its memory. When electronic coupon image 1 has been received from the user customized marketing service device, if the coupon use command is approved by the user for the abovementioned electronic coupon image 1 corresponding to shop 1, the abovementioned "electronic coupon image 1" received from the user customized marketing service device will be divided into 16 blocks. An electronic coupon image the same as electronic coupon image 1, which is stored in the memory in correspondence with shop 1, can be divided into 16 blocks.

Table 6. Method to Multiple Shops Data in the Memory

\begin{tabular}{|l|l|}
\hline Multiple shops & E-coupon image \\
\hline Shop 1 & E-coupon 1 \\
\hline Shop 2 & E-coupon 2 \\
\hline Shop N & E-coupon N \\
\hline
\end{tabular}

Thereafter, the pixel values of the 16 blocks of electronic coupon reference image 1 and those of the 16 blocks of electronic coupon image 1 can be compared with each other to distinguish blocks in which the last four bit values of the pixel value data were reversed from blocks in which the last four bit values of the pixel value data were not reversed among the 16 blocks of electronic coupon image 1 to find the bit values corresponding to the relevant blocks thereby extracting the 16bit electronic coupon code hidden in electronic coupon image 1. Thereafter, the mobile terminal of the user can display the extracted electronic coupon code on the screen to induce the user to use the electronic coupon by informing the relevant electronic coupon code seen from the screen to the worker at "shop1." This algorithm provides electronic coupon codes hidden in certain electronic coupon images to the user so that other users cannot forge or falsify the electronic coupon code thereby promoting fair operation of marketing services. 
Table 7. Codes Related to Coupon Transfer Function

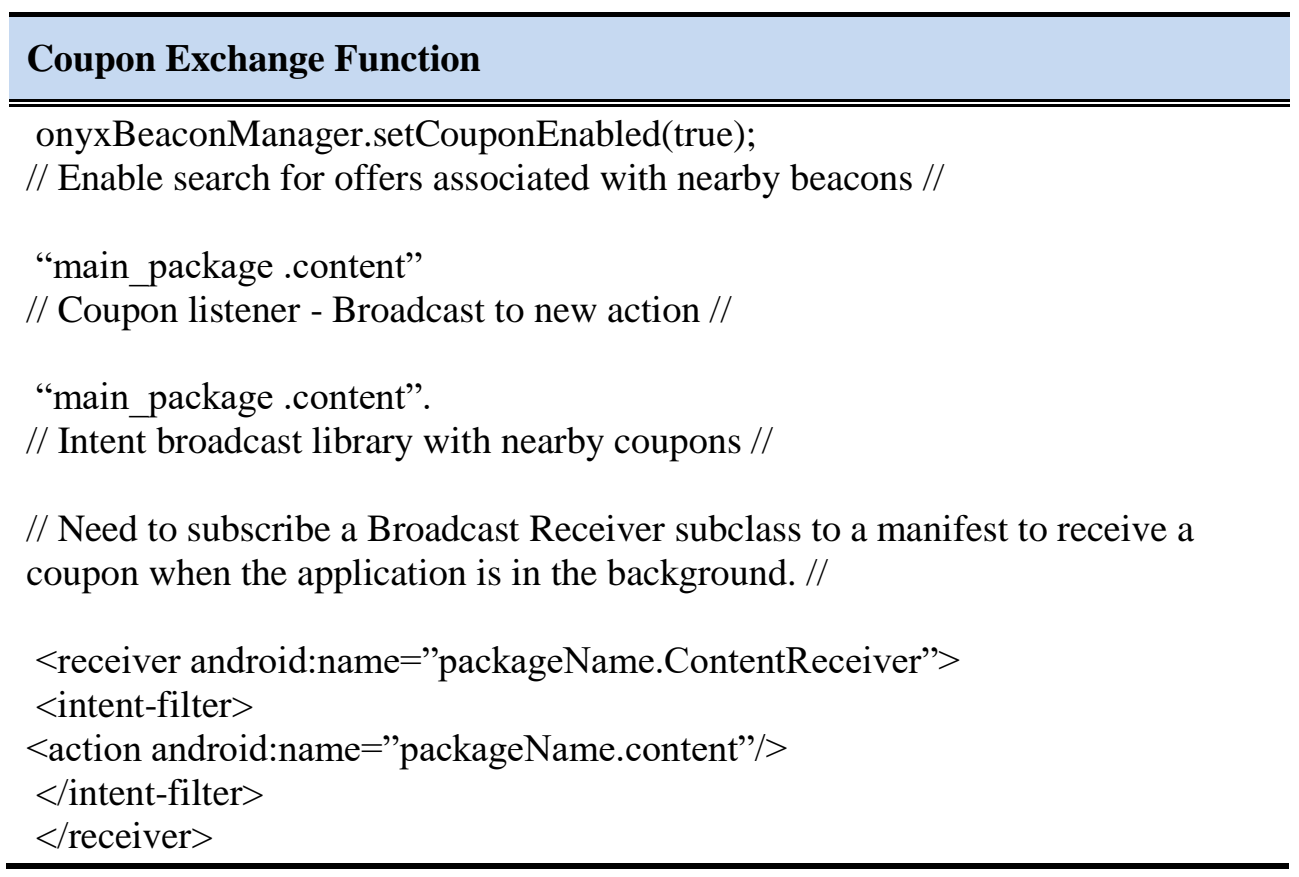

\section{Experimental Evaluation and Results}

In this study, for experimental evaluation of the beacon sensor device operation algorithm, first, 12 experimental target mobile devices of users corresponding to clients were set for four pilot test shops installed with five each beacon sensor devices as the targets of beacon sensor device performance experiments. In the experiment, when a user randomly approached one of the pilot test shops closely, the beacon sensor device installed at the relevant location created information identifying the user's visit to the relevant shop and transmitted the data to the main server data. In this regular method, 12 user mobile devices were put into contact with each shop three times. According to the results, each pilot test shop came into contact with users' mobile devices to create visit identifying information through users' unique ID identification information and transmitted the information as it was to the main server thereby collecting the data. In this case, it was identified that the visit identifying information receiving part entered into the beacon sensor device normally received the user information and that beginning with the contact of the count part with the first user mobile device, the number of times of receipt of user shop visit information from the five Beacon sensor devices at each pilot test shop was normally counted during the 'predetermined' period. As one of concrete results of the experiment, the point of time of the first contact with a user mobile device was "September 16, 2017", and thereafter, on "October 16" when one month passed, the fact that the user data information transmitted by the beacon sensor device, which received the relevant user mobile device information, was normally processed in the count part without any error, transferred to the main server, and sent to the information collection and storage place was identified. In the case of the e-coupon transmission part, the process through which at least one shop from which the number of times of receipt of customer visit information has been already counted to exceed the number of times of contact of the first user mobile device selected, was selected and e-coupons for products designated as contents for which coupons should be issued by the relevant shop were generated and transmitted to the mobile devices of the relevant users, was identified. 
Table 8. User Information Stored in the E-Coupon DB

\begin{tabular}{|l|l|l|}
\hline Pilot Test Shop & $\begin{array}{l}\text { Range of Average Residence } \\
\text { Time } \\
0 \sim 30 \text { minutes }\end{array}$ & $\begin{array}{l}\text { Information about the } \\
\text { Goods } \\
\text { Goods A }\end{array}$ \\
\cline { 2 - 3 } Shop No.1 & $30 \sim 60$ minutes & Goods B \\
\cline { 2 - 3 } & $60 \sim 90$ minutes & Goods C \\
\cline { 2 - 3 } Shop No.2 & $\ldots$ & $\ldots$ \\
\hline & $0 \sim 30$ minutes & Goods A \\
\cline { 2 - 3 } & $30 \sim 60$ minutes & Goods B \\
\cline { 2 - 3 } & $60 \sim 90$ minutes & Goods C \\
\cline { 2 - 3 } & $\ldots$ & $\ldots$ \\
\hline \multirow{2}{*}{$\ldots$} & $\ldots$ & $\ldots$ \\
\hline
\end{tabular}

In addition, resultant value indicating that the automatic transmission of the e-coupons for products sold by the shop particularly frequently visited by the users during a certain period among the pilot test shops to the users' mobile devices by the e-coupon transmission part enabled the operator of the relevant shop to increase profits and conduct continuous follow-up management of regular customers were obtained. In addition, information on different products that are targets of coupon transmission for different ranges of average stay times of experimental target users divided into multiple levels for individual shops in the database in charge of the coupon transmission part was normally stored indicating that sufficient data necessary for big data pattern analysis were successfully collected.

\section{Conclusion}

This study identified the fact that the improvement of operation algorithm with effective sensing and data transmission methods of low power Bluetooth based Beacon sensor devices will enable the collection of information on customers' shop visit patterns and analysis of the shop visit patterns with big data to transmit and provide customized ecoupons to customers, who are users, thereby providing opportunities for more efficient mobile marketing services to business operators and enabling the business operators to enjoy economic mutual effects through customized follow-up management of customers as well as the possibility of universalization of convergent contents that will enable pattern analysis regarding diverse customer information through integrated mobile marketing services with which users can experience diverse services in the Internet of Things (IoT) connected to the modern ubiquitous environments. This study can be said to be highly meaningful in that it prepared a moment to develop effective mobile marketing service techniques. In addition, many studies are considered necessary for marketing service techniques that will enable intensive analysis of customers visit and consumption patterns that change minute by minute and product trends and make the results into big data.

\section{Acknowledgments}

This research was supported by Basic Science Research Program through the National Research Foundation of Korea(NRF) funded by the Ministry of Science, ICT \& Future Planning(NRF-2017R1A2B4005185). 


\section{References}

[1] I. Demirkol, C. Ersoy and F. Alagoz, "MAC Protocols for Wireless Sensor Networks: A Survey", IEEE Communications Magazine, (2006) April.

[2] K. Kredo and P. Mohapatra, "Medium access control in wireless sensor networks", Computer Networks: The International Journal of Computer and Telecommunications Networking, (2007).

[3] K. Langendoen, "Medium Access Control in Wireless Sensor Networks", chapter in "Medium Access Control in Wireless Networks, Volume II: Practice and Standards", edited by H. Wu and Y. Pan, to be published by Nova Science Publishers, (2007).

[4] W. Ye, J. Heidemann and D. Estrin, "Medium Access Control with Coordinated, Adaptive Sleeping for Wireless Sensor Networks", IEEE/ACM Trans. on Networking, (2004).

[5] T. van Dam and K. Langendoen, "An Adaptive Energy-Efficient MAC Protocol for Wireless Sensor Networks", SenSys03, (2003).

[6] A. El-Hoiydi and J.-D. Decotignie, "Wise MAC: An Ultra Low Power MAC Protocol for Multi-hop Wireless Sensor Networks", ALGOSENSORS, (2004).

[7] El-Hoiydi, "Aloha with Preamble Sampling for Sporadic Traffic in Ad Hoc Wireless Sensor Networks", IEEE International Conference on Communications, (2002).

[8] P. Levis, S. Madden, J. Polastre, R. Szewczyk, K. Whitehouse, A. Woo, D. Gay, J. Hill, M. Welsh, E. Brewer and D. Culler, "TinyOS: An Operating System for Sensor Networks", Ambient Intelligence, (2005), pp. 115-148.

[9] K. Bouchard, R Ramezani, Arjun and A. Naeim, "Evaluation of Bluetooth beacons behaviour", IEEE 7th Annual Ubiquitious Computing, Electronics \& Mobile.

[10] P. Dickinson, G. Cielniak and O. Szymanezyk, "Indoor Positioning of Shoppers Using a Network of Bluetooth Low Energy Beacons", International Conference on Indoor Positioning and Indoor Navigation, (2016), pp. 1-8.

[11] S. Subedi, G.-R. Kwon, S. Shin, S. S. Hwang and J.-Y. Pyun, "Beacon Based Indoor Positioning System Using Weighted Centroid Localization Approach", Dept. Of Information and Communication engineering, Chosun Uni., (2016) August.

[12] R. Faragher and R. Harle, "Location Fingerprinting with Bluetooth Low Energy Beacons", IEEE Journal on Selected Areas in Communications, vol. 33, no. 11, (2015) November.

[13] Y. Wang, Q. Yang, G. Zhang and P. Zhang, "Indoor Positioning System Using Euclidian Distance Correction Algorithm with Bluetooth Low Energy Beacon", International Conference on Internet of Things and Applications (IOTA), (2016), pp. 243-247.

[14] N. Gung hyeon, L. il kwon and Lee Jaeg wang, "Using iBeacon positioning system location estimation techniques”, Journal of the Korea Institute of Information and Communication Sciences, vol. 19, no. 4, (2015).

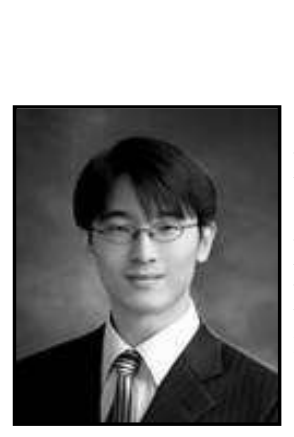

\section{Authors}

Seo-Ji Hoon, received his Bachelor degree in 2008 at Seoul National University of Science and Technology from department of Safety Engineering. He finished his MS and Ph.D. at Incheon National University from Department of Computer Science and Engineering in 2010 and 2015 respectively. His research interest includes Data Mining, Database Management and Sensor Networking.

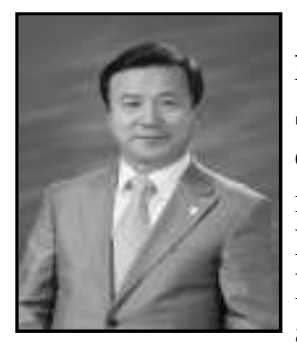

Choi-Jin Tak, received his B.S. degree in Mathematics and his M.S. degree in Computer Science from Dongkuk University, Seoul, Korea, in 1977 and 1982, respectively. He received Ph.D. degree in Electronics from Kyunghee University, Seoul, Korea, in 1991.Since 1987, he has been a Faculty Member at the Department of Computer Science of Incheon National University. His research interests include cryptography, database systems, and mobile and distributed computing 